\title{
Hydrogen Spillover Mechanisms over a Graphite Surface
}

\author{
Dmitry V.Leshchev ${ }^{1, *}$ \\ ${ }^{1}$ Center for Advanced Studies of Peter the Great St.Petersburg Polytechnic University, RU- 195251, St.-Petersburg, Russia
}

\begin{abstract}
Four different mechanisms of hydrogen spillower over carbon surface are considered in the framework of a single quantum-chemical approach. Two of them, described in the literature, are the physical migration of a bonded hydrogen atom along the surface and the edge of the graphene. One of the mechanisms proposed by the author does not include the migration of atomic hydrogen, but is realized via a transfer of carbon radical over a carbon sheet and generation of $\mathrm{H}$ atom in another place. Calculated activation energies of all four mechanisms are compared.
\end{abstract}

\section{Introduction}

Hydrogen spillover is a migration of the active hydrogen particles from the generation source (usually on noble metal clusters) to the reactive zone. Several mechanisms previously proposed in the literature for hydrogen spillover over the oxide surfaces are employed for such phenomena at the carbon surfaces, but all of them do not explain recently obtained experimental data [1,2] (which indicate the hydrogen spillover via a gas phase) and, in many cases, contradict the thermodynamic or chemical kinetic laws. However, when a spillover on the carbon surfaces is considered as a chain process between carbon and hydrogen radicals [3], these experimental data can be easily explained.

\section{Problem statement}

Two variants of the hydrogen spillover over a carbon surface are proposed in literature, confirmed by quantum-chemical modeling: migration of a charged [4] and neutral bounded particle [5]. The choice is made in favor of the migration of a charged particle, since the activation energy of this process is close to the experimental. However, the energy required for the formation of a proton is not taken into account, the processes of neutralization due to the electron transfer from a conducting graphite surface to a proton, as well as generation of the current in a conductor without an obvious energy source, are not considered at all. In [3] two variants of a hydrogen spillover over a graphite surface were proposed for the first time. Since it is difficult to compare the results of quantum-chemical calculations performed by different approaches, here various spillover mechanisms over carbon surfaces are compared within a single quantum-chemical approach: mechanisms with the migration of charged particles over the surface [4], a mechanism of hydrogen atom migration along the edge and the surface of graphite [5], the "relay" mechanism between hydrogen radicals and hydrogen molecules [3], the transfer of a carbon radical center and the new generation of a hydrogen radical [3]. Like any chain process, the last two mechanisms include a non-linear stage, when one act of atomic hydrogen generation leads to many acts of substitution in the product.

\subsection{Computational details}

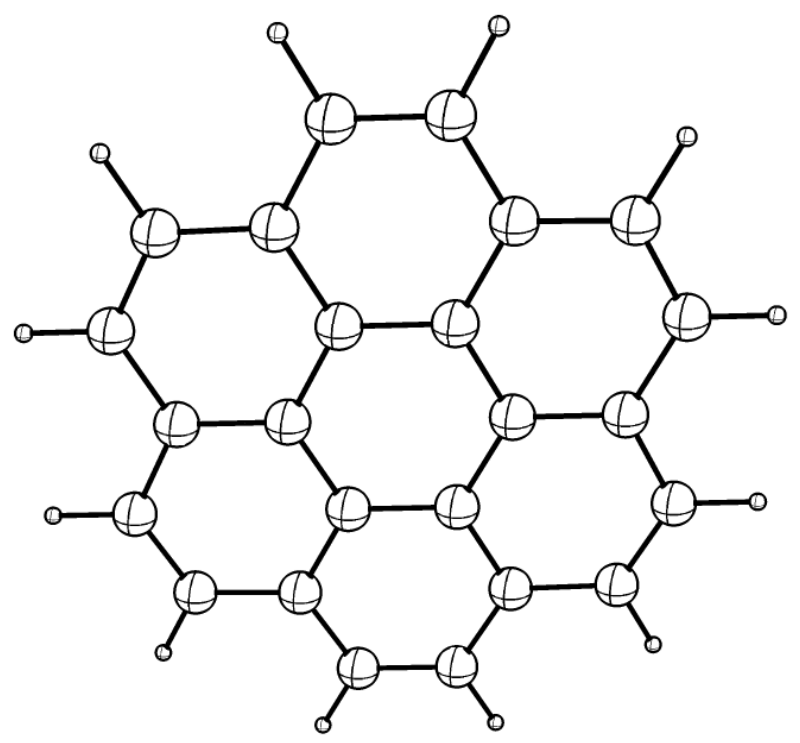

Fig. 1. The model of graphene cluster

The spillover of hydrogen atom on graphene was investigated using a seven-six-member ring carbon model as shown in Figure 1. Density functional theory B3LYP combined with the $6-31 \mathrm{G}+(\mathrm{d}, \mathrm{p})$ basis set was used in all cases. Full geometry optimizations and vibration analysis were performed at all stationary points to confirm their nature, namely the equilibrium point with no imaginary frequency or a transition state with one imaginary frequency whose mode corresponds to the 
reaction coordinate. In addition, intrinsic reaction coordinate calculations were performed for each transition state to verify its corresponding reactants and products. All calculations were done using the Gaussian 09 program.

\section{Results and discussion}

The following four sections discuss the details of the results obtained for the above mentioned 4 processes. The last section is devoted to the summary of obtained results and further work.

\subsection{Migration of charged particles}

Similarly to [4], the process of transfer of a hydrogen atom between neighboring carbon atoms and the same process for a charged system was considered, Fig. 2. As expected, in the case of a bound proton, the charge is distributed over all carbon and hydrogen atoms. The activation energies were 33.8 and $13.08 \mathrm{kcal} / \mathrm{mol}$, respectively. In contrast to [4], we obtained a negative enthalpy of attachment of atomic hydrogen to the central atoms of the cluster, which amounted to $-11.2 \mathrm{kcal} / \mathrm{mol}$ and $\Delta \mathrm{G}=-3.37 \mathrm{kcal} / \mathrm{mol}$, which shows that the reaction is favorable. For comparison, the energy of hydrogen detachment from a similar position from [5] is equal to $42 \mathrm{kcal} / \mathrm{mol}$. The enthalpy and $\Delta \mathrm{G}$ of proton addition are much larger and in our calculations were 190 and $182 \mathrm{kcal} / \mathrm{mol}$, respectively, which is close to the values obtained in [4]. However, the obtained activation energy for the hopping of a hydrogen atom from one surface carbon to another is much more than the binding energy of this atom with carbon; accordingly, the process of its cleavage and transition to the gas phase will be more likely than migration along the surface in a bound state.

Although the activation energy is close to the experimental value $15.5 \mathrm{kcal} / \mathrm{mol}$ [7], this spillover mechanism has significant drawbacks. Where does the energy for the proton formation come from? Unlike spillover over oxide surfaces, there are no oxidizing agents that would provide continuous oxidation of hydrogen. Another drawback of this approach is the incompatibility of the proton and conducting graphene: it is obvious that since the work function of graphite is much less than the hydrogen ionization potential, the electron will easily transfer from the carbon subsystem (or the metal subsystem associated with it) to the proton, and it will become a hydrogen atom, having lost all the advantages in energy and activation energy. This would explain the hydrogen spillover through the gas phase, but then the driving force of this process is not clear, since in general the metal catalyst decomposes molecular hydrogen into atoms in the gas phase, which should not happen from the point of view of thermodynamics.

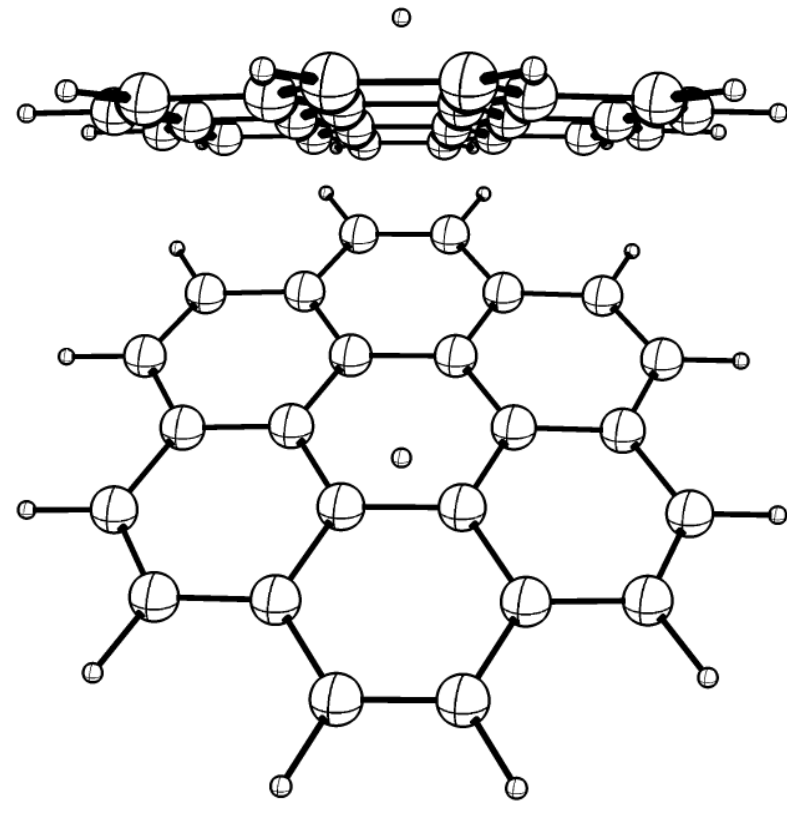

Fig. 2. The transition state of proton migration (side and top view)

\subsection{Migration on the edge of a graphite sheet}

For comparison, only reactions with minimal barriers were taken from [5]. Corresponding transition states are shown in Figure 3, a and b. The results are shown in Table 1. In contrast to [5], we considered also the transition reactions after the addition of two hydrogen atoms to the system. In that case the system contains an even number of electrons, and the activation energy increases sharply (Fig.4, a and b, Table 1). This suggests that this migration path is very sensitive to the attachment of a paired hydrogen atom, and a migration path along the edge will be blocked by creation of a saturated pair of neighboring carbon atoms. Since graphite has a finite number of edges, this spillover mechanism is limited in time.

Among the obvious drawbacks of this mechanism it can be mentioned a high activation energy (which according to the results of [5] and our calculations is greater than the hydrogenation energy) and the implicit reverse generation of the radical for the spiller through the gas phase or for reactions with the target.

\subsection{Radical transfer}

Carbon conjugate systems have the unique property of transferring the active center (dangling bond) from one end of system to another with a slight rearrangement of the geometry of the carbon skeleton. Thus, after the hydrogenation of one of the graphene atoms adjacent to the catalyst, the formed neighboring radical center can easily migrate along the sheet (according to our estimates the value of the electron translocalization barrier on the other atom is only $12 \mathrm{kcal}$, which is associated with a small difference in the geometries of $\mathrm{sp}^{3}$ of the radical center and $\mathrm{sp}^{2}$ carbon atom at the edge of the sheet). 
(1)
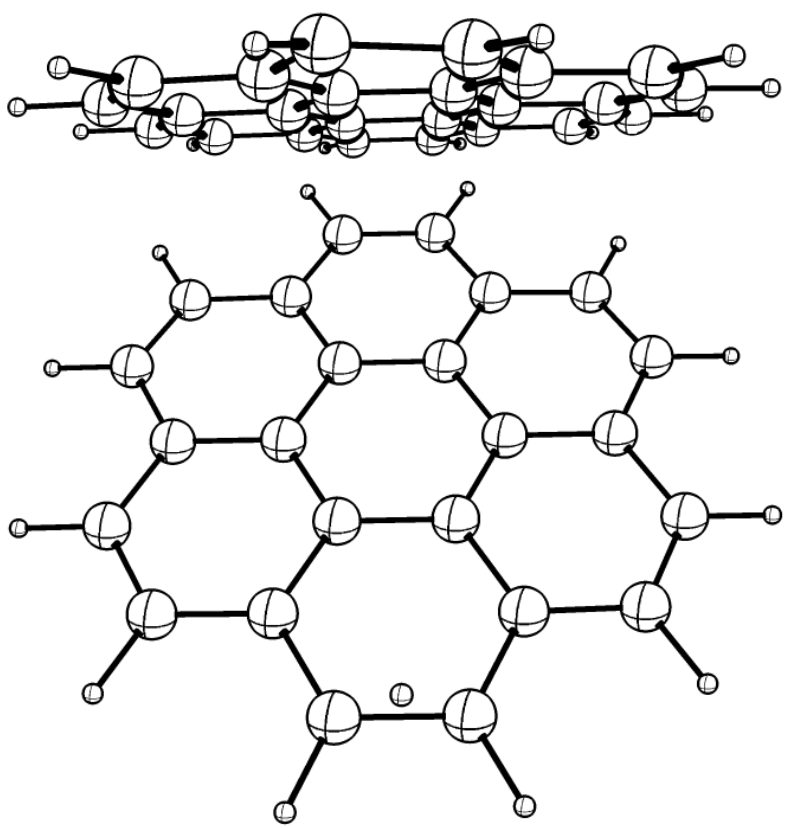

(a)
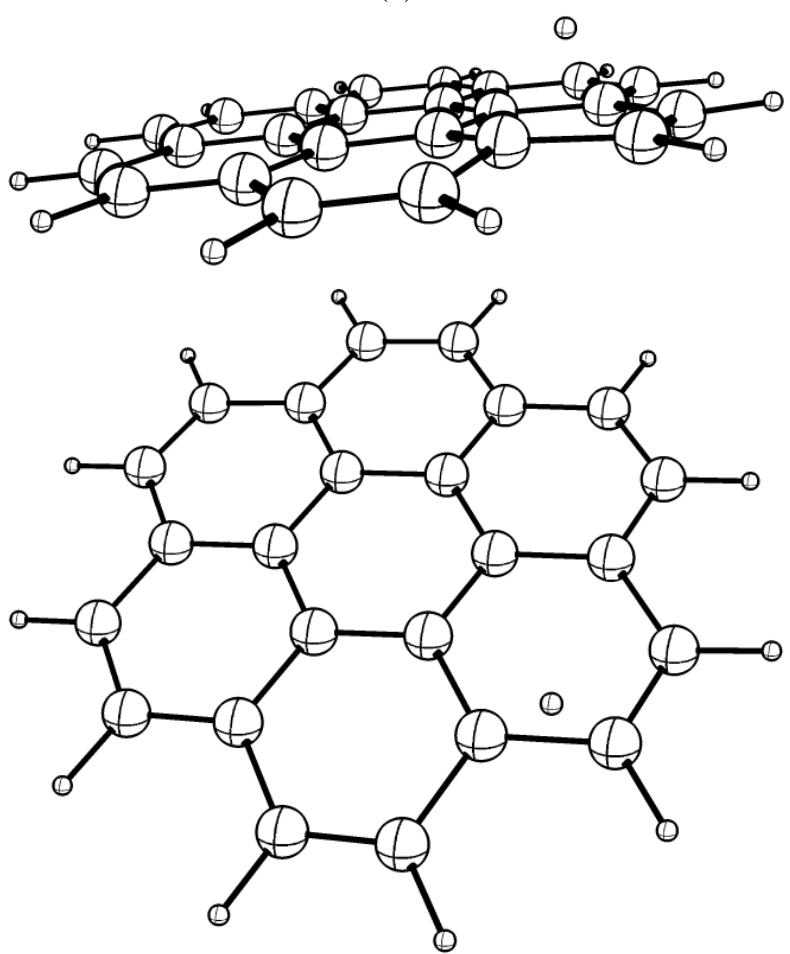

(b)

Fig. 3. The transition state of edge migration, a and $b$ for radicals (side and top view)
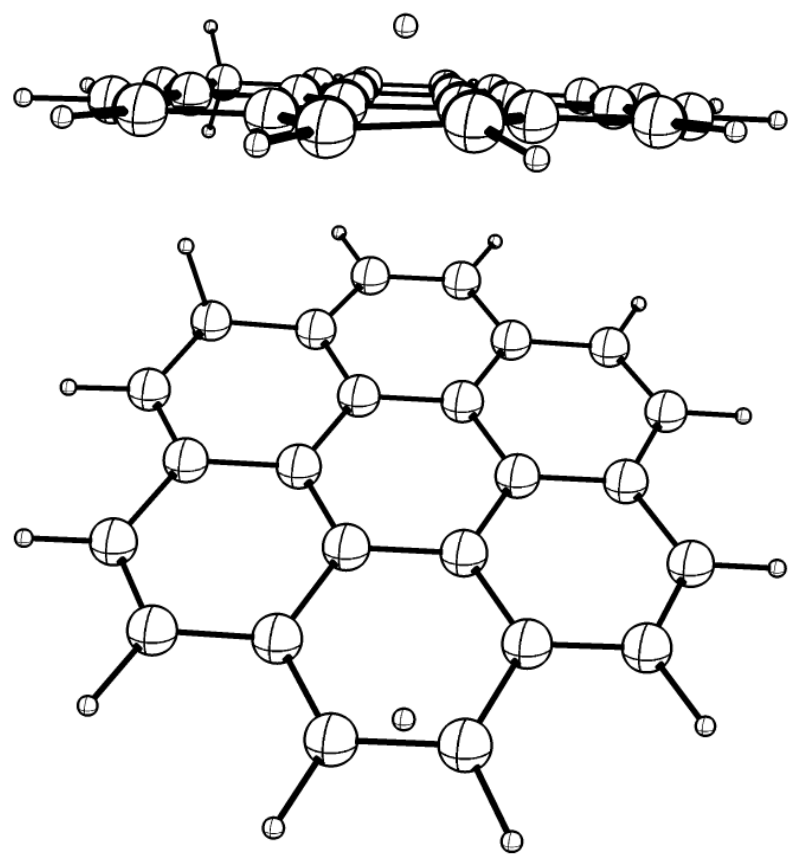

(a)
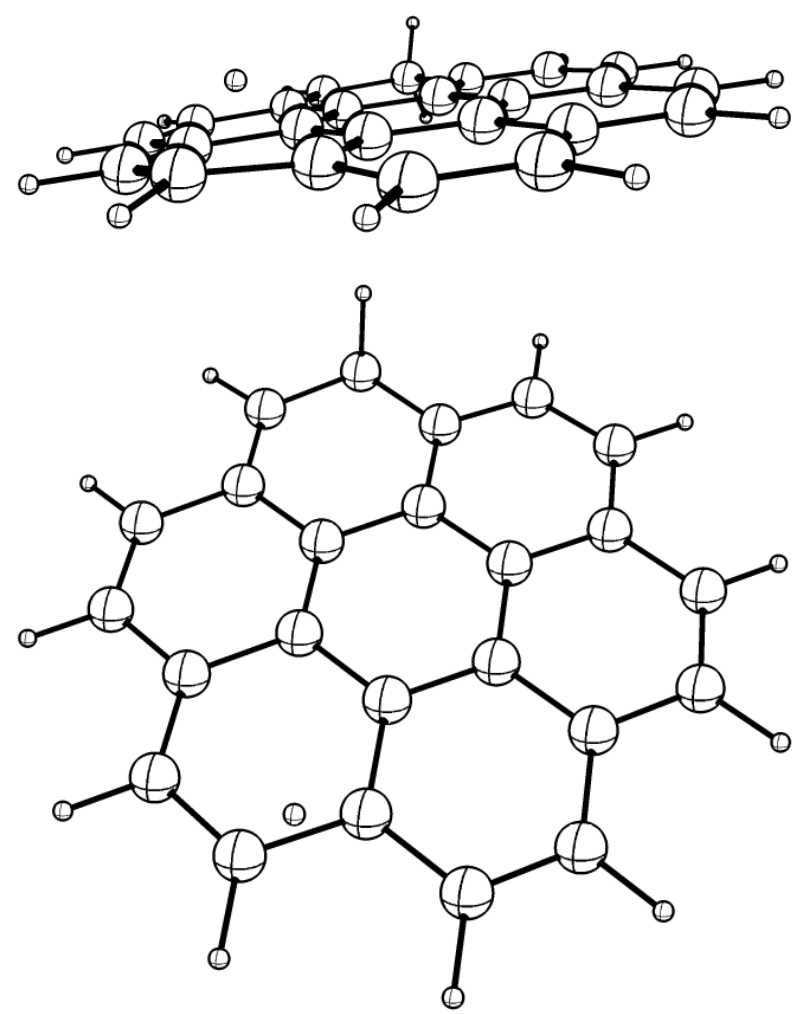

(b)

Fig. 4. The transition state of edge migration, $a$ and $b$ for non radicals (side and top view)

Like any other $\mathrm{sp}^{3}$ hybridized carbon radical, this atom can react with $\mathrm{H}_{2}$ and attach a hydrogen atom, generating atomic hydrogen, which remains in the gas phase. For aliphatic compounds corresponding activation energy is about $12 \mathrm{kcal} / \mathrm{mol}$; for aromatic compounds, it turns out to be slightly larger (for a newborn $\mathrm{sp}^{3}$ carbon). Thus, the hydrogen spillover is replaced by two 
hydrogenation reactions, one with $\mathrm{Ea}=0 \mathrm{kcal} / \mathrm{mol}$, the second with Ea from 20 to $60 \mathrm{kcal} / \mathrm{mol}$, depending on the place of attachment of the second hydrogen atom. For edge defects, as $\mathrm{CH}_{2}$ group, this value can be even closer to $12 \mathrm{kcal}$ and to the experimental value [7].

The main source of energy for the spillover in this mechanism is the hydrogenation energy of graphite, and due to this energy, molecular hydrogen partially dissociates to atoms.

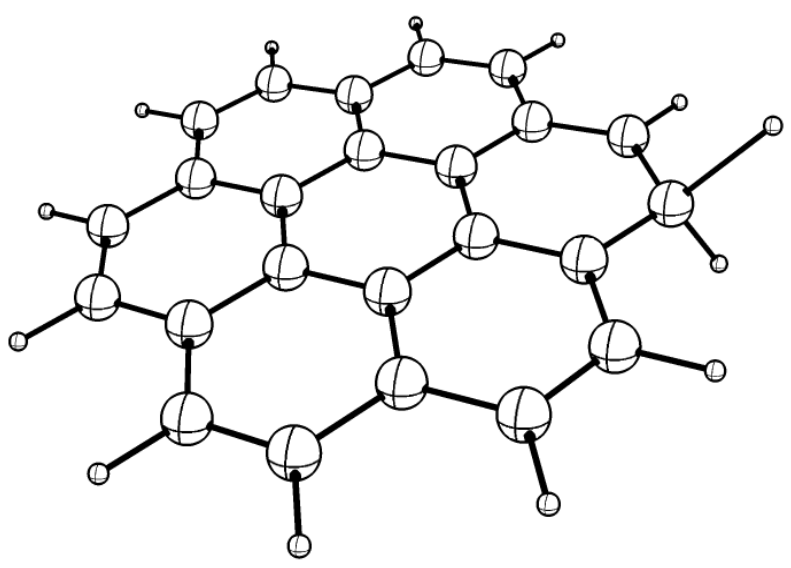

Fig. 5. Hydrogenation by $\mathrm{H}$

Table 1. Activation energies of reactions (in $\mathrm{kcal} / \mathrm{mol}$ )

\begin{tabular}{|l|l|l|}
\hline Reaction/Fig. & Ea, [Ref.] & Ea, this work \\
\hline $\begin{array}{l}\mathrm{H} \text { surface migration, } \\
\text { Fig.2 }\end{array}$ & - & 33.8 \\
\hline $\begin{array}{l}\mathrm{H}^{+} \text {surface migration, } \\
\text { Fig.2 }\end{array}$ & $15,5 / 10[4]$ & 13.08 \\
\hline $\begin{array}{l}\mathrm{H} \text { edge migration, Fig.3, } \\
\text { a }\end{array}$ & $47.5[5]$ & 42.1 \\
\hline $\begin{array}{l}\mathrm{H} \text { edge migration, Fig.3, } \\
\text { b }\end{array}$ & $48.9[5]$ & 45.2 \\
\hline $\begin{array}{l}\mathrm{H} \text { edge migration, Fig.4, } \\
\text { a }\end{array}$ & - & 56.1 \\
\hline $\begin{array}{l}\mathrm{H} \text { edge migration, Fig.4, } \\
\text { b }\end{array}$ & - & 58.2 \\
\hline \begin{tabular}{l}
$\mathrm{H}+\mathrm{H}_{2} \rightarrow \mathrm{H}_{2}+\mathrm{H}$ \\
\hline $\begin{array}{l}\text { Hydrogenation by H, } \\
\text { Fig.5 }\end{array}$
\end{tabular} & - & 3.55 \\
\hline $\begin{array}{l}\text { Hydrogenation by } \mathrm{H}_{2}, \\
\text { Fig.6, a }\end{array}$ & - & 0 \\
\hline $\begin{array}{l}\text { Hydrogenation by } \mathrm{H}_{2}, \\
\text { Fig.6, b }\end{array}$ & - & 19.7 \\
\hline
\end{tabular}

\subsection{Hydrogen relay}

This spillover mechanism is the simplest one. For its implementation, it is necessary to create a hydrogen atom physically adsorbed on graphene. As mentioned earlier, the $\Delta \mathrm{G}$ of the reaction of $\mathrm{H}$ atom with carbon, belonging to the center of the sheet, is only $-3.37 \mathrm{kcal} / \mathrm{mol}$ and, thus, this hydrogen exists both in a chemically adsorbed form and a physically adsorbed one. Such atomic hydrogen, as well as in the gas phase, can exchange with the hydrogen molecules, also adsorbed on the surface, and the hydrogen radicals easily migrate along the surface and then evaporate into the gas phase. On the other hand, atomic hydrogen reaching the edge of graphene can also react with the edge carbon atoms $(\Delta \mathrm{G}=-22 \mathrm{kcal} / \mathrm{mol})$. Corresponding carbon radical, formed on the outer edge, can react with $\mathrm{H}_{2}$ from the gas phase and generate atomic hydrogen in the gas, providing hydrogen spillover through the gas phase. Thus, these two last spillover mechanisms can move one into another, exchanging the carrier of the active center from atomic hydrogen to a carbon radical and vice versa.

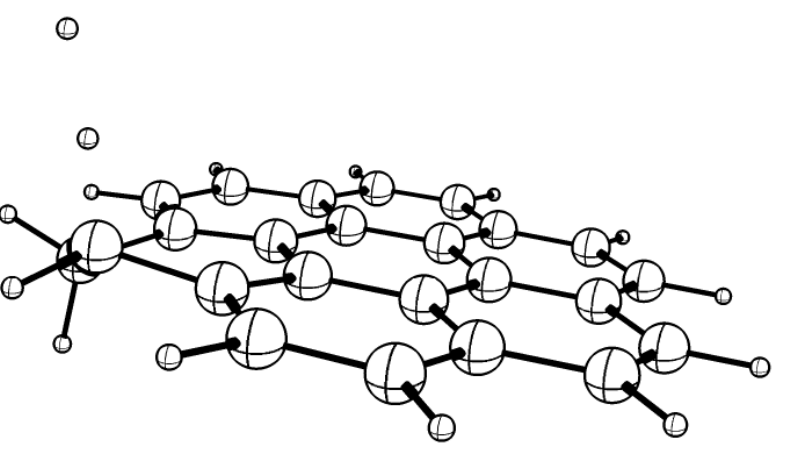

a

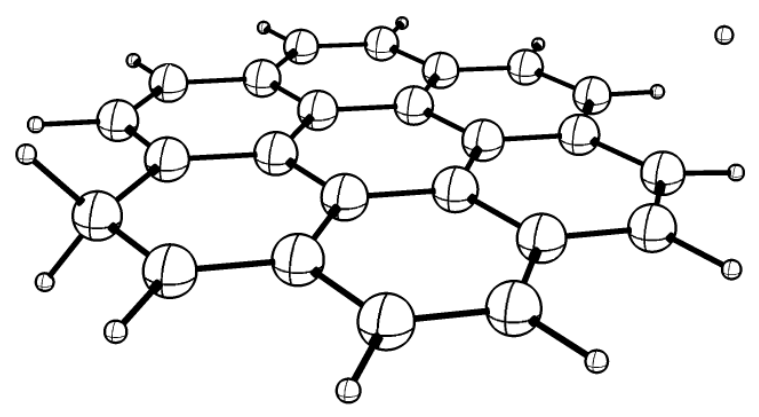

b

Fig. 6. Hydrogenation by $\mathrm{H} 2$ when attacking a neighboring atom (a) and the opposite edge of the cluster (b)

Table 2. The bond lengths characterizing transition states (in angstroms)

\begin{tabular}{|l|l|l|}
\hline Reaction/Fig. & C-H & H-H \\
\hline $\begin{array}{l}\mathrm{H} \text { surface migration, } \\
\text { Fig.2 }\end{array}$ & 1.17868 & - \\
\hline $\begin{array}{l}\mathrm{H}^{+} \text {surface migration, } \\
\text { Fig.2 }\end{array}$ & 1.2468 & - \\
\hline
\end{tabular}




\begin{tabular}{|c|c|c|}
\hline $\begin{array}{l}\mathrm{H} \text { edge migration, Fig.3, } \\
\mathrm{a}\end{array}$ & 1.28488 & - \\
\hline $\begin{array}{l}\mathrm{H} \text { edge migration, Fig.3, } \\
\mathrm{b}\end{array}$ & 1.45697 & - \\
\hline $\begin{array}{l}\mathrm{H} \text { edge migration, Fig.4, } \\
\mathrm{a}\end{array}$ & 1.14813 & - \\
\hline $\begin{array}{l}\mathrm{H} \text { edge migration, Fig.4, } \\
\mathrm{b}\end{array}$ & 1.1595 & - \\
\hline $\mathrm{H}+\mathrm{H}_{2} \rightarrow \mathrm{H}_{2}+\mathrm{H}$ & - & 0.9318 \\
\hline $\begin{array}{l}\text { Hydrogenation by } \mathrm{H}_{2} \text {, } \\
\text { Fig.6, a }\end{array}$ & 1.19036 & 1.16868 \\
\hline $\begin{array}{l}\text { Hydrogenation by } \mathrm{H}_{2} \text {, } \\
\text { Fig.6, b }\end{array}$ & 2.02664 & 1.41173 \\
\hline
\end{tabular}

\section{Summary}

Four spillover mechanisms over carbon materials are proposed. Generation of an active radical center is a common stage for all those mechanisms. Its source is an atomic hydrogen, which is formed via $\mathrm{H}_{2}$ dissociation on the metal. One hydrogen atom, after bonding with a carbon atom included in the conjugated system, forms a carbon radical. Then 1) the given hydrogen atom being in the bounded state can migrate along the surface or 2) along the edge of the sheet, or 3) the carbon radical can migrate and in the next hydrogenation by $\mathrm{H}_{2}$ produce atomic hydrogen, or 4) the physically adsorbed hydrogen atom can migrate along the surface. After migration, atomic hydrogen must leave the surface and be transported through the gas phase to react with the target, as shown in experiments with a hydrogen spillover through the gas phase [1,2]. For two first mechanisms, the question of the evaporation of atomic hydrogen into the gas phase remains open; in the last two, the evaporation is a natural part of the mechanism. After reaching with the target, atomic hydrogen (tritium) generates a chain of radical reactions leading to the replacement of hydrogen atoms of the substrate by tritium. If atomic hydrogen was able to reach graphite back, it can recombine with the previously attached atom, and then the system can return to its original state, except that the isotopic exchange on target takes place.

Since one reaction of hydrogen atom generation can correspond to a set of isotope exchange reactions registered in the experiments, the limiting stage can be the reaction with a substrate that is close to the experimental value and not a hydrogen spillover over a carbon surface. It is also possible that for large clusters the activation energy of the hydrogenation reaction by $\mathrm{H}_{2}$ is also less than a current value and is closer to the experimentally observed value.

The work compares these mechanisms by modeling the processes of transfer and calculating transition states. The analysis shows that the migration of the carbon radical most fully explains the experimental data and does not contradict thermodynamics. Further it is planned to continue the work in this direction, both by modeling the gas-dynamic aspects of the spillover through the gas phase and by quantum-chemical modeling of various variants of transition states and carbon radical transfer.

This work was partially supported by the Russian Foundation for Basic Research (project № 19-08-00533).

\section{References}

1. I.A. Razzhivina, G.A. Badun, S.B. Artemkina, M.G. Chernysheva, A.L. Ksenofontov, E.D. Grayfer, A.V. Garshev, Radiochemistry,61, 66 (2019)

2. I.A. Razzhivina, G.A. Badun, M.G. Chernysheva, A.V. Garshev, V.P. Shevchenko, K.V. Shevchenko, I.Yu. Nagaev, N.E. Shchepina, Mendeleev Comm., 26, 59 (2016)

3. D.V. Leshchev, FNCN, Special Issue (2020), (accepted for publication)

4. Y.A. Borisov, Y.A. Zolotarev, E.V. Laskatelev, N.F. Myasoedov, Russ.Chem.Bull., 46, 407 (1997).

5. H.T. Nguyen, L.K. Huynh, T.N. Truong, Carbon, 121, 248 (2017)

6. M.J. Cohen, N.C. Handy, R. Hernandez, W.H. Miller, Chem. Phys. Lett., 192, 407 (1992)

7. T. Fleisch, R. Aberman, J. Catal., 50, 268 (1977). 